\section{Microscopic Methods in Metals Edited by U. Gonser (Springer-Verlag, Berlin, 1986)}

This volume is the 40th in Springer-Verlag's Topics in Current Physics series. It consists of 12 individual chapters authored by experts in their respective fields and covers a wide range of so-called "microscopic" techniques which can be applied to the study of metals. In fact, most of these techniques are easily applicable to other forms of materials as well. The chapters are ordered, according to the editor, in a sequence corresponding to the decreasing length-scale to which they apply. For example, the first substantive chapter concerns scanning acoustic microscopy and therefore corresponds to acoustic wavelengths in the gigahertz frequency regime. Staying in the area of $\mathrm{mi}-$ croscopy, the next chapter concerns high-resolution electron microscopy; the third deals with field ion microscopy. In each of these chapters, as with the remaining chapters, the overview is enough that the nonspecialist can derive an extremely accurate impression of the method and its applicability; however, the detail is inadequate for the interested reader to actually utilize the method. This is explicitly acknowledged in the editor's introductory remarks. Each chapter includes several specific examples of the technique's application to various problems and is appropriately augmented by copious references for the reader interested in greater detail.

The chapter on acoustic microscopy emphasizes its utility for probing in depth in regions of a material that have variations in acoustic impedance and advertises the technique as being unique in that regard. In the area of electron microscopy, scanning electron microscopy is omitted in favor of a detailed description of transmission electron microscopy and its utility for determining structure at nearly an atomic scale. Field ion microscopy, less broadly applicable than electron microscopy, is treated in some detail. The chapter is augmented by a section on the atom probe where the additional possibility of identifying the constituents of the field emitter is indicated.

The fourth chapter deals with $x$-ray and neutron diffraction and treats diffraction phenomena in poly- and noncrystalline alloys. Topics ranging from small-angle scattering in grazing incidence $x$-ray scattering to structure of metallic glasses are used as examples.

The fifth chapter treats extended $x$-ray absorption fine structure (EXAFS). This chapter and the two succeeding chapters on x-ray photoemission spectroscopy (XPS) and Auger electron spectroscopy (AES) deal with phenomena best de- scribed as the interaction of the probing radiation with the individual atomic centers. These chapters begin with the fundamental principles of the technique and end with examples of applications. The natural emphasis, of course, for the EXAFS technique is on the determination of local structure; for the XPS technique, the determination of local chemical environment; and for the AES technique, the determination of composition.

The final five chapters deal with techniques somewhat less common than those described previously. First is positron annihilation. The chapter describes several types of measurements of annihilation of positrons in materials. This technique differs qualitatively from those previously described in that the probe (the positron) interacts with the lattice so that it is not obvious a priori what information is being revealed. The method's degree of indirectness complicates the interpretation of such data. Similarly, in the succeeding chapter on muon spectroscopy, it is made clear that one deduces characteristics of the sample by noting the effect of the environment on the polarization of the muon and its decay.

The last three of these chapters deal with nuclear physics based techniques. The first technique is perturbed angular correlation of nuclear radiation, in which excited nuclear states decay and the angular distribution of decay radiation can be related to the electric and magnetic interaction of the nuclear moments with their environment.

An extensive chapter on nuclear magnetic resonance then provides good grounding in the basic principles of the technique, the influence of magnetic and electric fields, and the various modalities of NMR experiments such as the spinecho method. The final chapter, written by the volume's editor, covers Mössbauer spectroscopy. After describing the fundamental origin of the technique, it treats the various types of interactions that affect the Mössbauer spectra.

As a whole, this volume may appear to be a combination of technique descriptions which do not fit well together. The span between acoustic microscopy and perturbed angular correlations is so wide that it is unlikely a single researcher would have a sincere interest in both. However, as a reference text which the materials researcher might consult for a summary of a technique, it is an excellent resource. Some characterization techniques which should have been included as microscopic techniques were omitted, and these include the various versions of ion scattering and electron resonance. However, this volume is recommended for researchers who would like to cursorily familiarize themselves with a tech- nique they have not previously used.

Reviewer: E.N. Kaufmann is leader of the Materials Division of the Chemistry and Materials Science Department at Lawrence Livermore National Laboratory.

\section{Rates of Phase \\ Transformations}

\section{R.H. Doremus}

\section{(Academic Press, 1985)}

This short 175-page book serves as a good basis for a graduate student's introduction to phase transformations. In fact, the book grew out of such a graduate student course at Rensselaer Polytechnic Institute. The author's emphasis on general principles also makes this book valuable to a wider range of materials scientists and others in related fields.

The author has succeeded in presenting a great deal of material succinctly, yet the book is very readable with good examples. Some of the brevity, however, is at the expense of detailed derivation and development of specific expressions.

The book contains eleven chapters. An all-too-brief introduction entitled "Thermodynamics" should probably have been expanded, although such material is liberally sprinkled throughout the text. Diffusion and phase changes and a very good treatment of the thermodynamics of interfaces follow. Several chapters deal with nucleation; e.g. liquid drops from vapor as well as from condensed phases, and an extension into phase separation of liquids.

The next several chapters involve crystal growth from vapor, solidification, and crystal growth from solution. The crystal growth chapters would have benefited from some discussion of the more recent work in semiconductor crystal growth and epitaxy; however, the general underlying concepts are there and the reader can branch from that point.

The final two chapters deal with grain growth and precipitation in metals.

All these chapters should equip graduate students or other informed readers with the important principles and background information on which to base more detailed study. Toward this end, each chapter includes a bibliography of important books, review articles, and research articles to help launch the reader deeper into his particular interests.

This volume is highly recommended to graduate students and active scientists and researchers interested in the kinetics of phase transformations. The succinct treatments with examples along with review references and research papers make this a good volume for a personal technical library.

Reviewer: Ralph Jaccodine is Sherman Fairchild Professor of Solid State Materials at Lehigh University. 